\title{
Quasi-State-Specific QCT Method for Calculating the Dissociation Rate of Nitrogen in Thermal Non-Equilibrium
}

\author{
S. Voelkel* and P. Varghese ${ }^{\dagger}$ \\ Department of Aerospace Engineering and Engineering Mechanics, \\ The University of Texas at Austin, Austin, TX, 78712 \\ V. Raman \\ Department of Aerospace Engineering, \\ The University of Michigan, Ann Arbor, MI, 48109
}

\begin{abstract}
The dissociation of nitrogen was studied using a quasi-classical trajectory (QCT) analysis in the context of calculating the dissociation rate surface for a dense range of temperatures for use in computational fluid dynamics (CFD) applications. By sampling rovibrational states from a Boltzmann distribution but uniformly sampling the relative speed, the dissociation rate was calculated for translational and rovibrational temperatures between $8000 \mathrm{~K}$ and $20000 \mathrm{~K}$. The justification for this approach was verified by analyzing different sampling techniques. It was found that uniformly sampling the relative speed increased the uncertainty of the thermally averaged dissociation rate, but the same QCT results could be used for a large range of temperatures. This is in contrast to Monte Carlo sampling techniques, where a new batch of trajectories must be simulated for each desired temperature. To generate the dissociation rate surface, 500 million trajectories were simulated, and the non-equilibrium rates were compared to other models and experimental data, generally showing good agreement.
\end{abstract}

\section{Nomenclature}

$b \quad$ Impact parameter, $\AA$

$E_{r v} \quad$ Quantized rovibrational energy

$f_{g} \quad$ Relative speed probability distribution function

$f_{r} \quad$ Rotational state probability distribution function

$f_{r v} \quad$ Rovibrational state probability distribution function

$f_{v} \quad$ Vibrational state probability distribution function

$g \quad$ Relative translational speed, $\AA /$ fs

$g_{s} \quad$ Spin degeneracy

$J \quad$ Rotational quantum number

$k_{B} \quad$ Boltzmann constant, $\mathrm{J} / \mathrm{K}$

$k_{d} \quad$ Thermal non-equilibrium dissociation rate, $\mathrm{cm}^{3} / \mathrm{s} / \mathrm{mol}$

$k_{e q}$ Thermal equilibrium dissociation rate, $\mathrm{cm}^{3} / \mathrm{s} / \mathrm{mol}$

$k_{\text {Park }}$ Park model dissociation rate, $\mathrm{cm}^{3} / \mathrm{s} / \mathrm{mol}$

$N \quad$ Total number of trajectories

$N_{d} \quad$ Number of dissociative trajectories

$P_{d} \quad$ Probability of dissociation

${ }^{*}$ Graduate Student, Department of Aerospace Engineering and Engineering Mechanics, Student Member, AIAA

${ }^{\dagger}$ Professor, Department of Aerospace Engineering and Engineering Mechanics, Senior Member, AIAA

${ }^{\ddagger}$ Associate Professor, Department of Aerospace Engineering, Senior Member, AIAA 
$Q_{r v}$ Rovibrational partition function

$Q_{v} \quad$ Vibrational partition function

$T$ Temperature, $\mathrm{K}$

$T_{t} \quad$ Translational temperature, $\mathrm{K}$

$T_{r} \quad$ Rotational temperature, $\mathrm{K}$

$T_{r v} \quad$ Rovibrational temperature, $\mathrm{K}$

$T_{v} \quad$ Vibrational temperature, $\mathrm{K}$

$v \quad$ Vibrational quantum number

$x \quad$ target function

$x i_{r} \quad$ Reactant rotational state

$x i_{t} \quad$ Reactant translational state

$x i_{v} \quad$ Reactant vibrational state

$\boldsymbol{\xi} \quad$ Reactant state vector

$\delta k_{d} \quad$ Standard deviation of the dissociation rate, $\mathrm{cm}^{3} / \mathrm{s} / \mathrm{mol}$

$\delta P_{d} \quad$ Uncertainty of the probability of dissociation

$\delta x \quad$ Uncertainty of the target function

$\delta \sigma \quad$ Uncertainty of the dissociation cross-section, $\AA^{2}$

$\eta \quad$ Normalization factor

$\mu \quad$ Reduced mass, $\mathrm{kg}$

$\tau \quad$ Reactant orientation vector

$\varphi \quad$ Efficiency function

$\sigma \quad$ Dissociation cross-section, $\AA^{2}$

$\zeta \quad$ Optimized Park model parameter

\section{Introduction}

Dissociation rates of nitrogen in a bath, i.e. $\mathrm{N}_{2}+\mathrm{N}_{2} \rightarrow \mathrm{N}_{2}+\mathrm{N}+\mathrm{N}$, at high temperatures with thermal non-equilibrium have been studied extensively due to interest in re-entry vehicle design. ${ }^{1-4}$ Computational fluid dynamics (CFD) codes integrate thermal nonequilibrium models into existing platforms through a variety of means, including multiple-temperature and state-specific models. For the re-entry problem, the conventional model is based on two temperatures, wherein the translational and rotational energy modes of the molecules are characterized by a temperature $T$, and the vibrational energy modes of the molecules are characterized by a vibrational temperature $T_{v}$. In each cell, the dissociation rate of nitrogen is calculated based on the local $T$ and $T_{v}$, which varies with both spatially and temporally. Thus, access to the full nonequilibrium dissociation rate surface, i.e. the dissociation rate over a range of $T$ and $T_{v}$ fields, is critical to achieving accurate computational results in a CFD calculation. This work presents a robust methodology for calculating the dissociation rate surface of $\mathrm{N}_{2}+\mathrm{N}_{2} \rightarrow \mathrm{N}_{2}+\mathrm{N}+\mathrm{N}$ and integrating the results alongside modern CFD programs.

For nitrogen dissociation, the most common approach to define the dissociation rate on a continuous field is by using Park's model. ${ }^{5}$ That is, for any $T$ and $T_{v}$, the dissociation rate is calculated using the conventional Arrhenius kinetics rate equation at an effective temperature, which is a combination of $T$ and $T_{v}$. A single parameter is used to define the effective temperature relation, which is determined based on a subset of directly calculated nonequilibrium rates. Because there is only one parameter to fit, only a small subset of the nonequilibrium rates calculated over wide range of $T$ and $T_{v}$ need to be directly calculated. The tradeoff for this simple model is global accuracy. Specifically, the relative impact of $T_{v}$ compared to $T$ on the rate is not constant for all $T$ and $T_{v}$.

In contrast to the fitting procedure described above, this work presents a robust approach for calculating the nonequilibrium rate surface using a combination of direct calculation and interpolation. First, the nonequilibrium rate is directly calculated for a coarse-scale grid of $T$ and $T_{v}$. Then, the nonequilibrium rate is interpolated from the original grid onto the fine-scale grid of $T$ and $T_{v}$. This new set is tabulated to be sufficiently dense so that in a CFD application, accurate nonequilibrium rates can be interpolated given any realized $T$ and $T_{v}$. There are three sources of error in this approach: (1) the uncertainty associated with directly calculating the nonequilibrium rate, (2) the interpolation error associated with defining the rate on a fine-scale grid, and (3) the interpolation error associated with defining the spatially and temporally local 
rate in a CFD simulation. In this work, the fine-scale grid is assumed to be sufficiently dense so that the error accrued during the CFD application is negligible. The correlation of errors (1) and (2) is nontrivial, but understanding this balance is critical to minimizing the global uncertainty of the nonequilibrium rate surface.

The nonequilibrium dissociation rate was numerically calculated using a quasi-classical trajectory (QCT) analysis. ${ }^{6}$ Such QCT studies have been used in previous studies. ${ }^{3,7}$ In QCT studies, the nuclei of a system are treated as classical particles driven by a potential energy surface (PES), and, in the case of nitrogen dissociation, bimolecular collisions are simulated. The initial conditions of the collision, denoted as a trajectory, are sampled based on specified energy states or from Maxwell-Boltzmann distributions characterized by temperature states, respectively denoted as state-specific and state-averaged sampling techniques. The dissociation rate given this state is then determined from the statistics associated with the sampled trajectories. Typically, many trajectories are required at a given state to ensure a small error in the computed rate. For a new state however, a new batch of trajectories must be simulated. As mentioned before, minimizing the variance (or uncertainty) associated with each rate versus directly calculating more rates (as opposed to using interpolation) is a balance when the full nonequilibrium rate surface is being tabulated.

This work is an extension of the work done by Candler and Truhlar's research groups to account for integration into CFD programs. As a brief summary, the global $\mathrm{N}_{4}$ potential energy surface was developed by Paukku et al. from approximately 17000 ab initio data points, ${ }^{2}$ and later improved by Bender et al. by introducing mixed exponential-Gaussian variables in the fit. ${ }^{7}$ These surfaces have been used in QCT studies to understand the effect of rovibrational nonequilibrium on the reaction rate. Bender et al. validated the thermal equilibrium rate and analyzed the dissociation rate at 13 points $T-T_{r v}$ space. Though not sufficient to define the full rate surface, the results showed that the $T_{r v}$ more dramatically affected the dissociation rate compared to $T$. More recently, Bender et al. decoupled the rotational and vibrational modes to calculate the rate versus $T$ and $T_{v}$, as described above. ${ }^{7}$ This work extensively analyzed the effect of the reactant state on different collision paths, including single dissociation, double dissociation, and nitrogen swapping. This work extends this previous work by developing a robust process for integrating QCT-derived rates into CFD applications and understanding the uncertainty associated with these rates.

In conjunction with the previously described objectives, this work also analyzed strategies associated with sampling the initial conditions and monitoring the uncertainty. First, a mode-independent sampling technique was implemented into a new massively-parallel QCT program. That is, the translational, rotational, and vibrational energy are all independently sampled a Maxwell-Boltzmann PDF, a uniform PDF, or from a set of predetermined values. A similar strategy was also developed for the impact parameter, which is another input for a trajectory. This allowed us to assess how different sampling techniques affect the uncertainty of the dissociation cross-section. For instance, the benefit of uniformly sampling the relative translational energy is that the rate can be calculated at any temperature, but, as will be presented below, the tradeoff is higher uncertainty. Also, an algorithm was developed for monitoring and controlling uncertainty in QCT calculations. In general, the dissociation cross-section has a direct correlation to the probability of dissociation for a specific state. But, for a single trajectory, the product state is typically a binary result: either the products were formed or not. For an infinitely large set of trajectories, the probability based on these binary results converges to a fixed value. For finite resources, this fixed value is never reached, but the variance of the present result can be estimated. Using this variance, an automation strategy was developed that dynamically adapts the QCT algorithm to ensure that the overall variance uniformly conforms to a user-defined tolerance.

After implementing these strategies, nonequilibrium dissociation rates for the reaction $\mathrm{N}_{2}+\mathrm{N}_{2} \rightarrow \mathrm{N}_{2}+\mathrm{N}+\mathrm{N}$ were calculated using a PES developed by Bender et al. ${ }^{7}$ The set of directly calculated rates was used in an interpolation scheme to define the rate surface on a fine-scale grid, tabulated for use in CFD applications. The balance between minimizing the variance of the directly calculated dissociation rates versus sampling more states was analyzed in the context of minimizing the global variance of the averaged dissociation rate surface. The remainder of this abstract is organized as follows. Section II discusses the formulation of the QCT model. Section A defines the the sampled PDFs, Section B describes the process of calculating the dissociation rate and its uncertainty, and C presents the QCT program features. Finally, Section III analyzes the results of this work. These results consist of two studies. The first study analyzes the dissociation rate uncertainty in the context of sampling techniques, and the second study calculates the dissociation rate for translational and rovibrational temperatures ranging from $8000 \mathrm{~K}$ to $20000 \mathrm{~K}$ using a quasi-state-specific sampling technique. From these results, the rates are validated by thermally averaging the data and comparing the results to 
other existing models and experimental data, the dissociation rate and efficiency function are analyzed in context of how dramatically the rovibrational and translational temperature affect the dissociation rate, and Park's two-temperature model is calibrated and compared to the non-equilibrium data.

\section{QCT Formulation}

The QCT methodology is derived from two main assumptions. First, from quantum mechanics, the BornOppenheimer approximation assumes that the nuclear and electron states of the system are decoupled. ${ }^{8}$ From this assumption and by ab initio methods, the eigenvalues of the electronic Schrödinger equation are (numerically) calculated for a fixed nuclear position. These eigenvalues correspond to the potential energy of the system, and when defined on the whole space of nuclear configurations, this energy acts as a continuous function, aptly referred to as the potential energy surface (PES). This surface is differentiable almost everywhere, and the only isolated non-differentiable points being where two or more nuclei from the system have the same global position. Second, the classical assumption extends the Born-Oppenheimer approximation and assumes that not only are the nuclear and electron motions decoupled, but that the nuclei can be treated as classical particles ${ }^{6}$ so the wave function associated with each nucleus is assumed to be a delta function. The driving force between the nuclei is defined as the negative derivative of the aforementioned PES.

Solving the classical equations of motion for a set of nuclei is the main process in a QCT simulation. Computationally, this amounts to solving a set of ordinary differential equations (ODE) representing the evolution of the phase space of the nuclei for a variety of initial conditions and analyzing the outcomes. The numerical ODE solver chosen for this work was the adaptive Runge-Kutta Prince-Dormand $(8,9)$ method as implemented in the open source GNU Scientific Library (GSL). ${ }^{9}$ Though the method is not symplectic, the error tolerance was set sufficiently low so that the total energy of the system did not drift noticeably over a typical trajectory time frame.

Regarding the initial conditions of the ODE system, the reactants' state bounds the initial phase space of the nuclei in the system. For this work, multiple state definitions have been implemented. In short, the translational, rotational, and vibrational energy of the reactants are prescribed based on this state, which in turn restricts the relative position and velocity of the reactants. The orientation of each reactant is sampled from a uniformly distributed sphere and the separation of the reactants was chosen to be greater than $15 \AA$ (the maximum separation recognized for this particular PES). ${ }^{2}$ The final specification is the impact parameter $b$ : the distance component between the reactants perpendicular to the relative motion. In this work, the maximum impact parameter $b_{\max }$ was set to $8.0 \AA$ based on the previous analysis by Bender et $a .^{3}$

\section{A. State Probability Distributions}

To calculate the thermally averaged dissociation rate, i.e. the rate as a function of one or more temperatures, the PDFs associated with the energy modes of the molecules needs to be defined. The relative translational energy PDF is given by

$$
f_{g}\left(g ; T_{t}\right)=\left(\frac{\mu}{2 \pi k_{B} T_{t}}\right)^{3 / 2} 4 \pi g^{2} e^{-\mu g^{2} / 2 k_{B} T_{t}}
$$

where $g$ is the relative translational speed, $T_{t}$ is a translational temperature, $\mu$ is the reduced mass of the reactant pair, and $k_{B}$ is the Boltzmann constant. The rovibrational energy PDF as a function of the rovibrational quantum numbers $v$ and $J$, and characterized by a rovibrational temperature $T_{r v}$, is given by

$$
f_{r v}\left(v, J ; T_{r v}\right)=\frac{g_{s}(J)(2 J+1) e^{-\left(E_{r v}(v, J)-E_{r v}(0,0)\right) / k_{B} T_{r v}}}{Q_{r v}\left(T_{r v}\right)},
$$

where $E_{r v}$ is the quantized rovibrational energy, $g_{s}$ is the spin degeneracy, and $Q_{r v}$ denotes the partition function. In re-entry vehicle simulations, typically, the rovibrational states of the nitrogen in the system after the bow shock are decoupled so that the vibrational and rotational states are characterized by $T_{v}$ and $T_{r}$, respectively. Such a decoupling procedure is not well-defined, and the derivation presented by Bender et 
$a l .{ }^{7}$ a vibration-prioritized framework, was used. Here, the rovibrational PDF is given by

$$
f_{r v}\left(v, J ; T_{v}, T_{r}\right)=\frac{f_{v}\left(v ; T_{v}\right) f_{r}\left(J ; T_{r} \mid v\right)}{\eta\left(T_{v}, T_{r}\right)},
$$

where $f_{v}$ is the vibrational PDF, $f_{r}\left(J ; T_{r} \mid v\right)$ is the rotational PDF conditioned based on the vibrational state, and $\eta$ is a normalization factor. In this form, the PDFs are defined by

$$
\begin{aligned}
f_{v}\left(v ; T_{v}\right) & =\frac{e^{-\left(E_{r v}(v, 0)-E_{r v}(0,0)\right) / k_{B} T_{v}}}{Q_{v}\left(T_{v}\right)} \\
f_{r}\left(J ; T_{r} \mid v\right) & =\frac{g_{s}(J)(2 J+1) e^{-\left(E_{r v}(v, J)-E_{r v}(0,0)\right) / k_{B} T_{r}}}{Q_{r v}\left(T_{r}\right)} \frac{1}{f_{v}\left(v ; T_{r}\right)} .
\end{aligned}
$$

Bender et al. ${ }^{7}$ have shown that the normalization factor varies between 0.95 and 1.10 for a large range of $T_{v}$ and $T_{r}$.

\section{B. Dissociation Rate Calculation}

Let the translational state $\xi_{t}$ denote a specific relative translational speed or a temperature characterizing the distribution of the relative translational speed, and let $\xi_{r}$ and $\xi_{v}$ analogously describe the rotational and vibrational state. Then, for $N$ randomly sampled trajectories from the phase space restricted by the reactants' initial state, denoted by $\boldsymbol{\xi}=\left(\xi_{t}, \xi_{r}, \xi_{v}\right)$, and an impact parameter $b$, the mean probability of dissociation is defined by integrating the probability of reaction over the initial orientation of the reactants, i.e.

$$
P_{d}(\boldsymbol{\xi}, b)=\int_{\boldsymbol{\tau}} f_{\boldsymbol{\tau}}(\boldsymbol{\tau}) P_{d}(\boldsymbol{\xi}, b, \boldsymbol{\tau}) d \boldsymbol{\tau},
$$

where $\boldsymbol{\tau}$ is the initial orientation vector and $f_{\boldsymbol{\tau}}$ is the PDF associated with the initial orientation. In regards to notation throughout this work, please note that if a dependency is dropped, i.e. $P_{d}(\boldsymbol{\xi}, b)$ instead of $P_{d}(\boldsymbol{\xi}, b, \boldsymbol{\tau})$, it is implied that the function has been averaged over the dropped variable. Using Monte Carlo integration, Eq. (6) reduces to

$$
P_{d}(\boldsymbol{\xi}, b)=\lim _{N \rightarrow \infty} \frac{N_{d}(\boldsymbol{\xi}, b)}{N(\boldsymbol{\xi}, b)},
$$

where $N_{d}$ is the number of trajectories resulting in nitrogen dissociation. The uncertainty of $P_{d}$, denoted by $\delta P_{d}$, is defined as two standard standard deviations from the mean, which, once again using Monte Carlo integration theory, reduces to

$$
\delta P_{d}(\boldsymbol{\xi}, b)=2 \frac{N_{d}(\boldsymbol{\xi}, b)}{N(\boldsymbol{\xi}, b)}\left(\frac{N(\boldsymbol{\xi}, b)-N_{d}(\boldsymbol{\xi}, b)}{N_{d}(\boldsymbol{\xi}, b)(N(\boldsymbol{\xi}, b)-1)}\right)^{1 / 2} .
$$

As the number of sampled trajectories goes to infinity, the uncertainty goes to zero and the subsequent dissociation rate converges to a fixed value. If no dissociative trajectories are recorded, then the uncertainty is zero, which may occur when the initial state corresponds to a low dissociation probability. Thus, by selecting the number of trajectories to simulate for a given state, the user is also defining the minimum observable probability, i.e. $P=1 / N$, which has a corresponding uncertainty of $\delta P=2 / N$. If a relative level of convergence per state is desired, trajectories are simulated at a particular state until $\delta P / P$ reaches a threshold. Using this approach, the number of trajectories per state will increase significantly for low probability states. Instead, an absolute level of convergence per state was utilized in this work so trajectories are simulated at a particular state until $\delta P$ reaches a threshold. The threshold is set to a value sufficiently low so that $N_{d}>1$ for all states, which was determined from preliminary calculations. This ensured that both low and high probability states converge uniformly, thus minimizing the global uncertainty of the probability for all states.

The dissociation cross-section at state $\boldsymbol{\xi}$ is calculated by integrating $P$ along $b$ weighted by $2 \pi b$, i.e.

$$
\sigma(\boldsymbol{\xi})=\int_{0}^{b_{\max }} 2 \pi b P_{d}(\boldsymbol{\xi}, b) d b .
$$


Because the integral is a linear operator, the uncertainty of the cross-section $\delta \sigma$ is defined by replacing $P$ with $\delta P$ in Eq. (9). For uniform sampling, the impact parameter space is discretized into $N_{b}$ cells, and the cross-section is the sum of the average values for each cell, i.e.

$$
\sigma(\boldsymbol{\xi})=\sum_{i=1}^{N_{b}}\left(\int_{b_{i-1 / 2}}^{b_{i+1 / 2}} 2 \pi b P_{d}(\boldsymbol{\xi}, b) d b\right) \approx \sum_{i=1}^{N_{b}} 2 \pi b_{i} P_{d}\left(\boldsymbol{\xi}, b_{i}\right) \Delta b_{i},
$$

where $P_{d}\left(\boldsymbol{\xi}, b_{i}\right)$ denotes the probability sampled from all impact parameters in the $i^{\text {th }}$ cell, and $\Delta b_{i}$ is the cell width. Note that if the impact parameter is sampled based on Monte Carlo integration, then Eq. (9) simplifies to

$$
\sigma(\boldsymbol{\xi}) \approx \pi b_{\max }^{2} P_{d}(\boldsymbol{\xi})
$$

The dissociation rate is defined by integrating the cross-section over the relative translational speed weighted by the Maxwell speed PDF characterized by a translational temperature $T_{t}$, i.e.

$$
k_{d}\left(T_{t}, \xi_{r}, \xi_{v}\right)=\int_{0}^{\infty} g f_{g}\left(g ; T_{t}\right) \sigma\left(g, \xi_{r}, \xi_{v}\right) d g .
$$

Rather than integrating from zero to infinity, the upper and lower bounds of $g$ are chosen so that $\chi(g)$ is approximately zero beyond these points. As before, the uncertainty of the dissociation rate $\delta k_{d}$ is defined by replacing $\sigma$ with $\delta \sigma$ in Eq. (12). If $\xi_{t}=g$ and $g$ is uniformly sampled, the relative translational speed is discretized into $N_{g}$ cells, and the dissociation rate is the sum of the average values for each cell, i.e.

$$
k_{d}\left(T_{t}, \xi_{r}, \xi_{v}\right)=\sum_{i=1}^{N_{g}}\left(\int_{g_{i-1 / 2}}^{g_{i+1 / 2}} g f_{g}\left(g ; T_{t}\right) \sigma\left(g, \xi_{r}, \xi_{v}\right) d g\right) \approx \sum_{i=1}^{N_{g}} g_{i} f_{g}\left(g_{i} ; T_{t}\right) \sigma\left(g_{i}, \xi_{r}, \xi_{v}\right) \Delta g_{i}
$$

where $\sigma\left(g_{i}, \xi_{r}, \xi_{v}\right)$ denotes the probability sampled from all relative translational speeds in the $i^{\text {th }}$ cell, and $\Delta g_{i}$ is the cell width. If $\xi_{t}=T_{t}$, then the cross-section is approximated by Monte Carlo integration, i.e.

$$
k_{d}\left(T_{t}, \xi_{r}, \xi_{v}\right) \approx\left(\frac{8 k_{B} T_{t}}{\pi \mu}\right)^{1 / 2} \sigma\left(T_{t}, \xi_{r}, \xi_{v}\right)
$$

where $k_{B}$ is the Boltzmann constant, and $\mu$ is the reduced mass of the reactant pair.

If $\xi_{v}$ and $\xi_{r}$ represent specific quantum numbers, i.e. $\xi_{v}=\left(v_{1}, v_{2}\right)$ and $\xi_{r}=\left(J_{1}, J_{2}\right)$, then the thermally averaged dissociation rate is calculated by averaging over the Boltzmann PDF characterized by vibrational and rotational temperatures $T_{v}$ and $T_{r}$, respectively. For the rovibrationally state-specific reaction

$$
\mathrm{N}_{2}\left(v_{1}, J_{1}\right)+\mathrm{N}_{2}\left(v_{2}, J_{2}\right) \longrightarrow \mathrm{N}_{2}+\mathrm{N}+\mathrm{N}
$$

both reactant's initial rovibrational state are independently averaged. So, the thermally averaged dissociation energy is given by

$$
k_{d}\left(T_{t}, T_{r}, T_{v}\right)=\sum_{\left(v_{1}, J_{1}\right)} \sum_{\left(v_{2}, J_{2}\right)} f_{r v}\left(v_{1}, J_{1} ; T_{v}, T_{r}\right) f_{r v}\left(v_{2}, J_{2} ; T_{v}, T_{r}\right) k_{d}\left(T_{t}, v_{1}, v_{2}, J_{1}, J_{2}\right)
$$

where the summations are over the set of valid rovibrational states, and though each reactant state has been independently averaged, $T_{v}$ and $T_{r}$ are the same for both reactants.

\section{Computational Details}

Alongside this work, a new QCT program was developed to take advantage of large-scale computational infrastructure. This program was heavily influenced by the VENUS program ${ }^{10}$ and the work of Bender et $a l .{ }^{3}$ The parallelization of the QCT algorithm is straightforward because each trajectory is independent of the other trajectories. However, due to the variation in processor speed and trajectory computational cost, a load balancing algorithm was necessary to maintain uniform computational load over all processors for the duration of a QCT simulation. Furthermore, for adaptive algorithms that dynamically adjust the simulation 
based on the intermediate uncertainty calculations, a communication infrastructure was necessary so that each processor core knew which cross-sections still needed to be calculated or improved.

These computational objectives were achieved by utilizing a single, master nodes that operates alongside a set of worker nodes. During the QCT initiation processes, the master node determines the necessary states to calculate the desired set of reaction rates. Then, during the online portion of the simulation, each worker node contacts the master node one-by-one for instructions, which is the status of the QCT simulation and the next state to analyze. Once these directions are received by the worker node, it simulates a batch of trajectories sampled from the given state. Afterwards, it recontacts the master node for further instructions, and the cycle continues. Thus, interprocessor communication is minimized because there is a central node in charge of all the directives.

For up to 4,096 cores (the most tested to date), this process computationally efficient. Figure 1 shows a preliminary parallel scaling assessment of the computational time for running five million trajectories on varied numbers of cores.

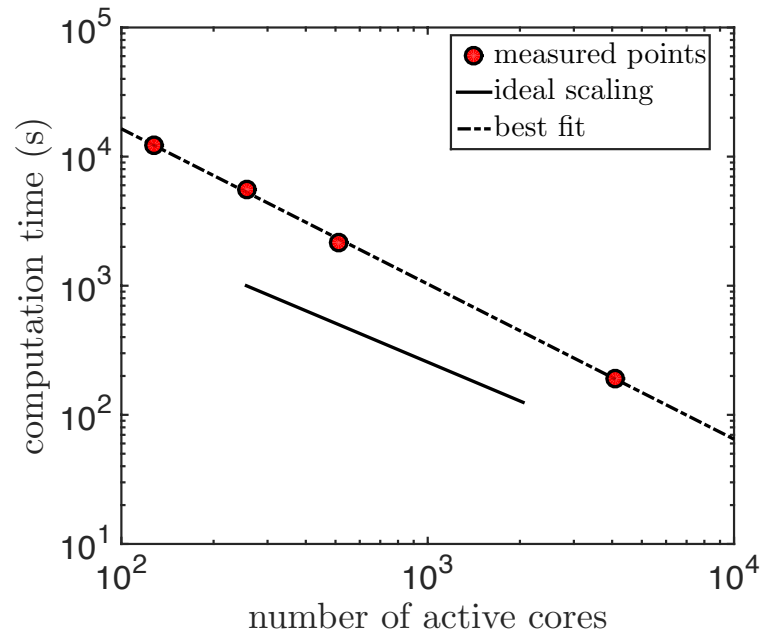

(a) Computational time versus the number of active cores

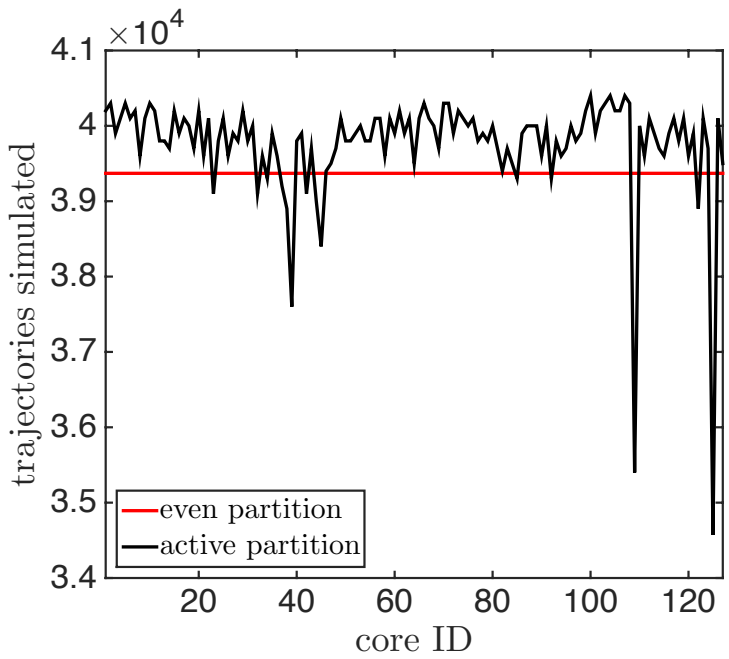

(b) Partition of trajectories among 128 cores

Figure 1: Parallel scaling assessment of QCT program for five million trajectories simulated using a varied number of cores

These results show that the code is both efficient, approximately linearly scaling with cores, and predictable, a small-scale simulation can be used to predict the computational cost of a large-scale simulation. Note that the perception that this scaling is more efficient than ideal is due to the to marginalized cost of the master node as the total number of cores increases. The active partition in Figure $1 \mathrm{~b}$ shows that in general, each cores simulates approximately the same number of trajectories, but a few of the trajectories run significantly fewer trajectories. To minimize the cost, the other cores balance this by simulating more trajectories. If an even partition was used, the slow cores would significantly increase the total simulation time.

Periodically throughout the online portion of the QCT simulation, at a time when the master node is not communicating with the worker nodes, the mater node calculates the set of cross-sections and their associated uncertainties from the results gathered up to that point. If the uncertainty of any cross-section is sufficiently low, below a user-defined absolute or relative tolerance, then the master node marks that state as complete. When all the states are complete, the master node communicates to the worker nodes that the job is complete. Due to the intricacies of post-processing the data for a variety of different sampling techniques and desired data points, this process is left offline and run as a separate program.

The interpolation strategy implemented for computing the nonequilibrium rate surface on a fine-scale grid of $T$ and $T_{v}$ was the multivariate interpolation and regression (MIR) scheme proposed by Wang et al. ${ }^{11}$ The scheme creates a high-order approximation function constructed from a set of data points on a scattered domain where the estimate of the approximation error at each data point is minimized. The set of data 
points, or target functions, used to create the interpolant were given by

$$
\begin{aligned}
x_{i} & =\log _{10}\left(k_{d}\left(T_{i}, T_{v, i}\right)\right) \\
\delta x_{i} & =\log _{10}\left(k_{d}\left(T_{i}, T_{v, i}\right)+\delta k_{d}\left(T_{i}, T_{v, i}\right)\right)-\log _{10}\left(k_{d}\left(T_{i}, T_{v, i}\right)\right),
\end{aligned}
$$

for $i=1, \ldots, N_{x}$ and $N_{x}$ is the number of rates directly calculated. The target function was chosen as $f=\log _{10}(k)$ because of its expected near-linear relationship with $T$ and $T_{v}$. Currently, the interpolation of the rate onto the fine-scale $T-T_{v}$ grid is done in a post-processing step. However, it is possible to integrate this process alongside the QCT program so that the surface can be dynamically checked throughout the simulation. However, because each directly calculated rate is converged within a user-defined uncertainty tolerance, this step was not implemented for this work.

\section{Results}

The results of this work are focused on the nonequilibrium dissociation rate of nitrogen in a bath, i.e. $\mathrm{N}_{2}+\mathrm{N}_{2} \rightarrow \mathrm{N}_{2}+\mathrm{N}+\mathrm{N}$. The analytical PES used in this work was developed by Bender et al., ${ }^{7}$ which was an extension of the surface developed by Paukku et al. from a set of approximately 17000 ab initio data points. $^{2}$

\section{A. Study I: Optimal Sampling Techniques}

The first study consisted of three test cases designed to analyze the effect of different sampling techniques on the estimated uncertainty. The goal of each test was to calculate the dissociation rate and estimate the corresponding uncertainty for six temperatures ranging from $10000 \mathrm{~K}$ to $20000 \mathrm{~K}$. One million trajectories were simulated per temperature, leading to a total of six million trajectories per case. The differences between each case were how the impact parameter and relative translational speed were sampled.The rovibrational states were sampled from a Boltzmann distribution at the corresponding temperatures. Table 1 shows the different PDFs used to sample the initial conditions for each test case.

\begin{tabular}{|c|c|c|c|}
\hline Test Case & $f_{b}(b)$ & $f_{g}(g ; T)$ & $f_{r v}(v, J ; T)$ \\
\hline 1 & Linear & Maxwell & Boltzmann \\
2 & Uniform & Maxwell & Boltzmann \\
3 & Uniform & Uniform & Boltzmann \\
\hline
\end{tabular}

Table 1: PDFs used to sample the initial conditions for each test case

Case 1 corresponds to full Monte Carlo sampling, so the cross-section and dissociation rate are given by Eq (11) and Eq (14), respectively. For case 2, the cross-section and dissociation rate are given by Eq (10) and Eq (14), respectively. For case 3, the cross-section and dissociation rate are given by Eq (10) and Eq (13), respectively. For the cases 2 and 3, the impact parameter was discretized into 32 cells ranging from $0 \AA$ to $8 \AA$. For case 3 , the relative translational speed was discretized into 25 cells. The minimum sampled speed was $10^{-4}$ as likely as the most probable speed based on the Maxwell PDF at $10000 \mathrm{~K}$, and the maximum sampled speed was $10^{-8}$ as likely as the most probable speed based on the Maxwell PDF at $20000 \mathrm{~K}$.

First, the effect of the impact parameter sampling technique was analyzed by comparing cases 1 and 2 . Figure 2 shows the probability of dissociation multiplied by $2 \pi b$ versus the impact parameter at $14000 \mathrm{~K}$ and $20000 \mathrm{~K}$. 


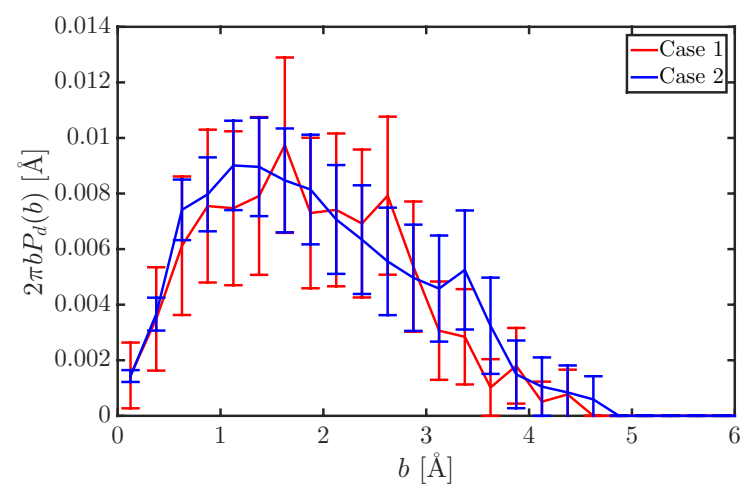

(a)

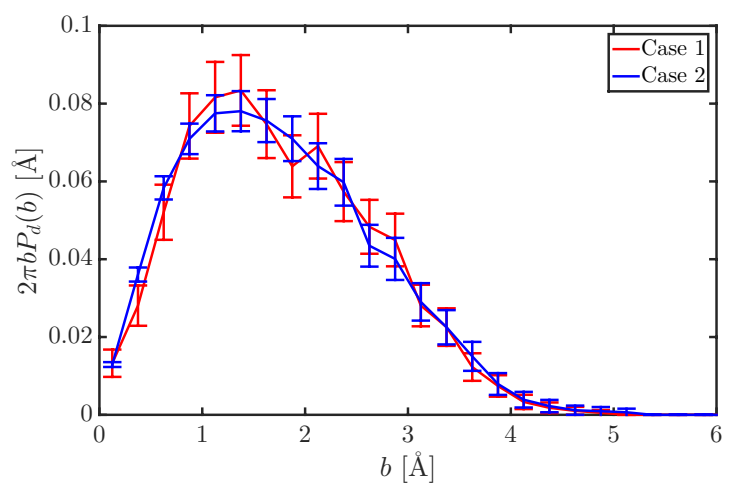

(b)

Figure 2: Probability of dissociation multiplied by $2 \pi b$ versus impact parameter: (a) $T=14000 \mathrm{~K}$, and (b) $T=20000 \mathrm{~K}$.

Note that the area under each curve is the corresponding cross-sections. Based on qualitative inspection, it is clear that as the temperature increases, the uncertainty decreases. This trend follows from the uncertainty definition in Eq. (8). The average uncertainty of the dissociation probability $\left\langle\delta P_{d}\right\rangle$ was normalized by the average dissociation probability $\left\langle P_{d}\right\rangle$ to create a quantified measure of comparison. The results are tabulated in Table 2.

\begin{tabular}{|c|c|c|}
\hline Test Case & $T[\mathrm{~K}]$ & $\left\langle\delta P_{d}\right\rangle /\left\langle P_{d}\right\rangle$ \\
\hline 1 & 14000 & 0.427 \\
2 & 14000 & 0.292 \\
\hline 1 & 20000 & 0.143 \\
2 & 20000 & 0.098 \\
\hline
\end{tabular}

Table 2: Probability distribution functions sampled from for each test case

For both low and high temperatures, the uniform sampling technique (case 2) has a lower average uncertainty compared to the linear sampling technique (case 1). Moving from case 1 to case 2 , at $14000 \mathrm{~K}$ the relative uncertainty decreased by $13.5 \%$, and at $20000 \mathrm{~K}$ the relative uncertainty decreased by $4.5 \%$. For case 1, too few trajectories were sampled for low impact parameters, where dissociation is more likely to occur, resulting in a higher variance. For instance, for one million sampled trajectories at $14000 \mathrm{~K}$, only 348 trajectories resulted in dissociation. However, when uniformly sampling, 1265 trajectories resulted in dissociation at $14000 \mathrm{~K}$. At high impact parameters, case 1 has a lower uncertainty compared to case 2, but the probability of reaction in these regions is already very small. Thus, the difference is not sufficient to improve the average relative uncertainty significantly.

One of the main drawbacks of both cases 1 and 2 are that for each desired translational temperature, a new batch of trajectories needs to be calculated. To account for this, test case 3 uniformly samples both the impact parameter and relative translational speed. With this in mind, Figure 3 shows the computed dissociation rate for each case. 


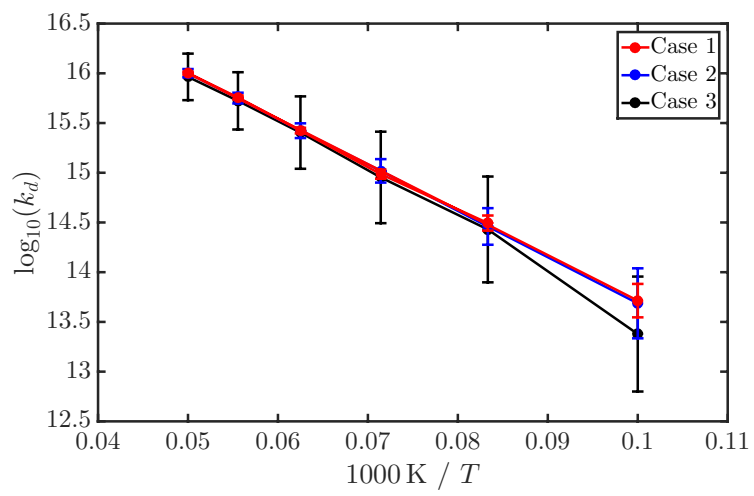

Figure 3: Thermal equilibrium dissociation rate versus the temperature. The units for $k_{d}$ are $\mathrm{cm}^{3} / \mathrm{s} / \mathrm{mol}$.

Notice that the dissociation rate's uncertainty for case 1 is now lower than for case 2, which is unexpected because it contradicts the results in Figure 2 and Table 2. This suggests that though Monte Carlo integration does not accurately capture low-level information, e.g. the probability versus the impact parameter, it instead, by assuming the PDF of the state, more accurately captures the high-level information, e.g. the reaction rate. This claim is also supported by the results for case 3, which has the highest uncertainty. However, for case 1 and 2, only 6 cross-sections were calculated, whereas for case 3, 150 cross-sections were calculated. For each cross-section in case 1 and 2, one million trajectories were simulated, but for case 3, only forty thousand trajectories were simulated per cross-section. This is the reason the uncertainty is so large for case 3. However, by uniformly sampling the relative translational speed, the dissociation rate at any translational temperature is calculated by averaging the speed-dependent cross-section weighted by the Maxwell PDF. Thus, because the uncertainty is not too large for case 3, it should be used to calculate the dissociation rate surface because more data points can be generated on a fine-scale grid of $T_{t}$ to create the interpolant.

A sampling concern with case 3 is that the rotational and vibrational energy sampling techniques are still state-averaged based on $T_{r}$ and $T_{v}$, respectively. But, for $\mathrm{N}_{2}+\mathrm{N}_{2}$, each reactant has approximately 9,200 bound and quasi-bound rovibrational states, and for both reactants together, there are almost 85 million total rovibrational state combinations. Thus, systematically sampling the rovibrational quantum state makes this problem computationally intractable. Even for a sparse selection of states, interpolation between the states adds a large amount of uncertainty. So, rather than sampling rovibrational quantum numbers, we sample a much smaller selection of rovibrational temperatures and interpolate the values in between. This feasibility of this process was confirmed in the second study.

Finally, the convergence of the three test cases was analyzed. Figure 4 shows the convergence of dissociation rate by plotting the uncertainty in the dissociation rate versus the number of trajectories sampled. 


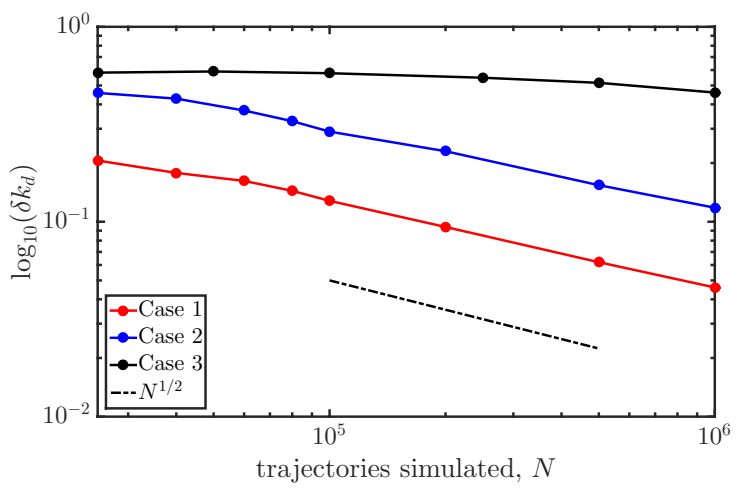

(a)

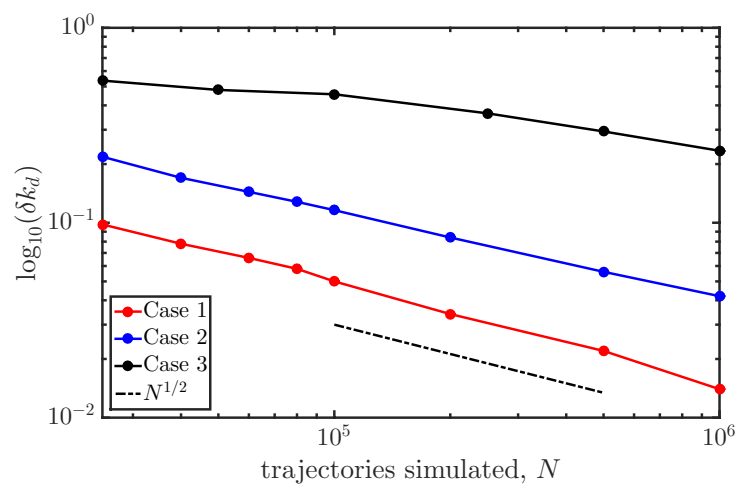

(b)

Figure 4: Convergence of dissociation rate based on estimated uncertainty for varied number of total trajectories: (a) $T=14000 \mathrm{~K}$, and (b) $T=20000 \mathrm{~K}$.

Cases 1 and 2 converge at a rate proportional to $N^{1 / 2}$, which corresponds to the expected rate for Monte Carlo integration. For case 3, the convergence rate is slower, especially at lower temperatures. Too few trajectories are simulated for each cross-section. Once a sufficient number of trajectories that result in reaction are simulated, especially for low-speed cross-sections, the convergence rate will increase. This is seen in Figure $4 \mathrm{~b}$, wherein case 3 converges at approximately the same rate as the other cases because a sufficient number of reactive trajectories have been simulated.

\section{B. Study II: Non-Equilibrium Dissociation Rate Surface Calculations}

The second study was a single run designed to calculate 400 unique cross-sections. That is, 100 evenly spaced relative translational speeds were studied for four rovibrational temperatures: $8000 \mathrm{~K}, 10000 \mathrm{~K}, 13000 \mathrm{~K}$, and $20000 \mathrm{~K}$. A total of 500 million trajectories were simulated on 2,048 cores over 15 hours. After the simulation, the dissociation rate was calculated at 66 translational temperatures evenly spaced between 8,0000 $\mathrm{K}$ and $25,0000 \mathrm{~K}$ for each of the rovibrational temperatures. These dissociation rates were used in the open source MIR library developed by Wang et al. ${ }^{11}$ to calculate the rate surface, as shown in Figure 5a.

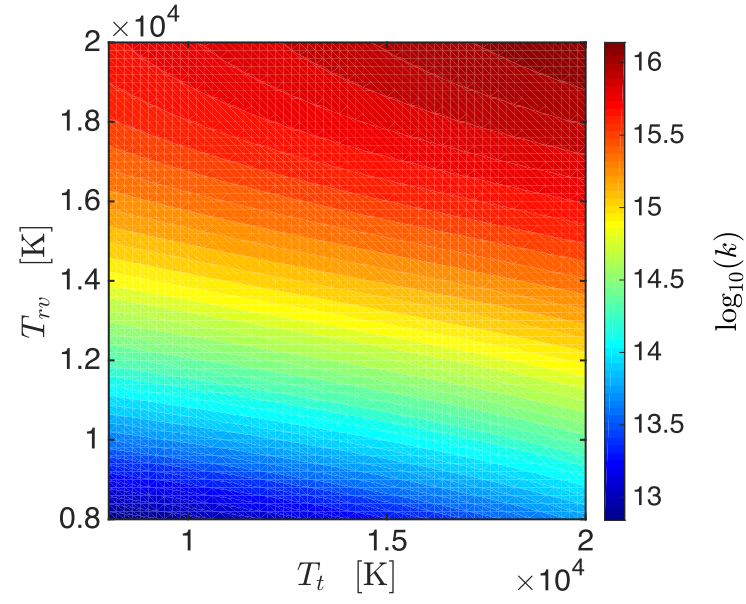

(a)

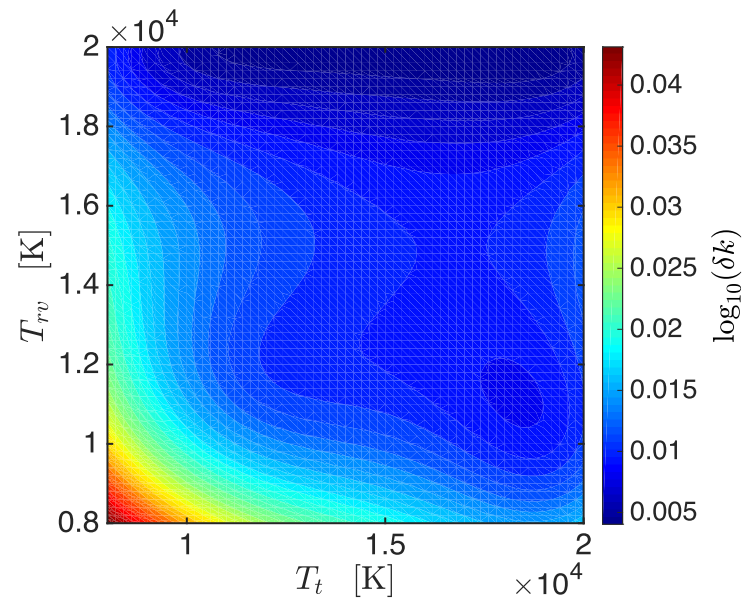

(b)

Figure 5: Dissociation rate versus translational and rovibrational temperature from the quasi-state-specific QCT method: (a) the dissociation rate surface, and (b) the standard deviation of the dissociation rate. The units for $k$ and $\delta k$ are $\mathrm{cm}^{3} / \mathrm{s} / \mathrm{mol}$. 
From this figure, it is clear that the rovibrational temperature has a significantly stronger effect on the dissociation rate than the translational temperature. The results by Bender et al. also follow this trend. $^{3}$ The uncertainty associated with the rate surface, which is predicted by the MIR scheme, is shown in Figure 5b. Looking at the the values between $T_{r v}=8000 \mathrm{~K}, 10000 \mathrm{~K}, 13000 \mathrm{~K}$ and $20000 \mathrm{~K}$, we observe that the uncertainty of the interpolated dissociation rates is most affected by the calculated rate's local uncertainty. The largest uncertainty is for low temperatures, specifically near $8000 \mathrm{~K}$ for both $T_{t}$ and $T_{r v}$. As with the previous study, this information can be dynamically analyzed during the QCT simulation to decide where more trajectories should be run to improve the global accuracy of the surface.

As validation, the slice from Figure $5 \mathrm{a}$ where $T_{r v}=T_{t}$, i.e. the thermal equilibrium rate, is compared with other published rates for nitrogen dissociation. The uncertainty bars correspond to two standard deviations from the mean, or a $95 \%$ confidence interval.

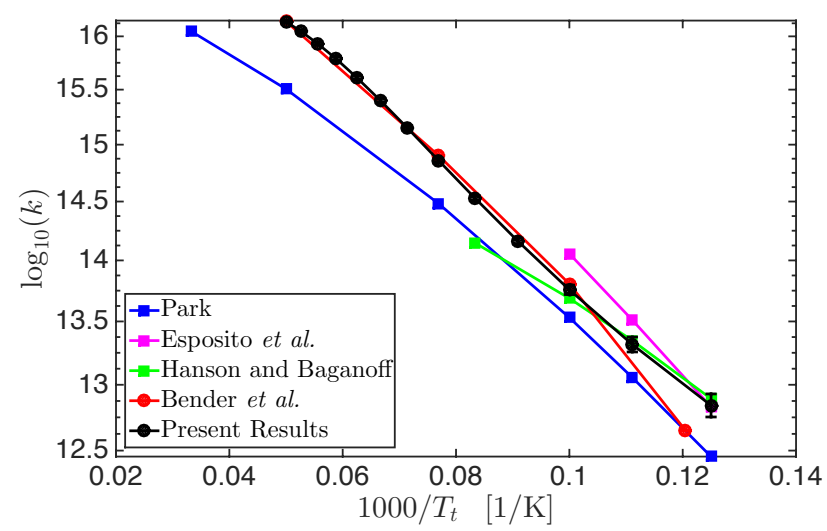

Figure 6: Thermal equilibrium dissociation rate versus the translational temperature $\left(T_{r v}=T_{t}\right)$. The units for $k$ are $\mathrm{cm}^{3} / \mathrm{s} / \mathrm{mol}$.

At low temperatures, the present results match, within the uncertainty bars, both the model from Esposito et al. calculated using the Park factor ${ }^{1}$ and shock-tube experimental studies of Hanson and Baganoff. ${ }^{12}$ From $8000 \mathrm{~K}$ to $10000 \mathrm{~K}$, the rate is very close to the results of Hanson and Baganoff. Above $10000 \mathrm{~K}$ the two diverge, but from $10000 \mathrm{~K}$ to $20000 \mathrm{~K}$, the present results closely match the rate calculated by Bender et al. ${ }^{3}$ The rate never exactly matches the rate from Park's model, ${ }^{5}$ but the two are close from $9000 \mathrm{~K}$ to $12000 \mathrm{~K}$. It should be noted that Park's model explicitly assumes $T_{r}=T_{t} \neq T_{v}$, whereas we have used $T_{r}=T_{v} \neq T_{t}$. We made this choice to compare our results directly with the results of Bender et al. ${ }^{3}$

From the dissociation rate surface in Figure 5a, the efficiency function is defined as the relative effectiveness of $T_{r v}$ in increasing or decreasing the dissociation rate, ${ }^{13}$ given as

$$
\varphi=\frac{k_{d}\left(T_{t}, T_{r v}\right)}{k_{e q}\left(T_{t}\right)}
$$

where $k_{e q}\left(T_{t}\right)=k_{d}\left(T_{t}, T_{r v}=T_{t}\right)$. The logarithm of the efficiency function for $T_{t}$ and $T_{r v}$ ranging from $8000 \mathrm{~K}$ to $20000 \mathrm{~K}$ is shown in Figure 7. 


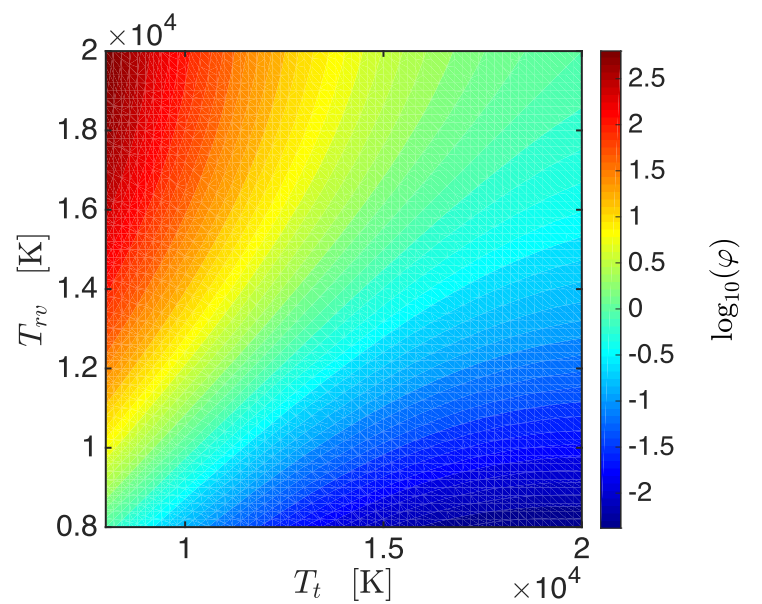

(a)

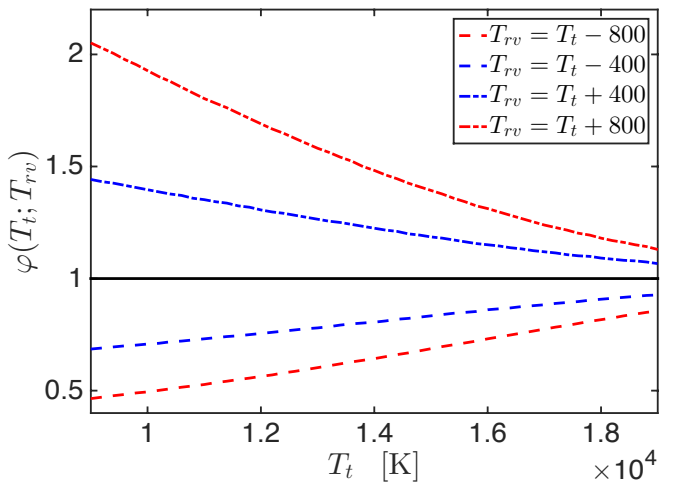

(b)

Figure 7: Efficiency function versus translational and rovibrational temperature from the quasi-state-specific QCT method: (a) the efficiency function surface, and (b) slices along $T_{r v}=T_{t}+\Delta T$.

The surface shows that for $T_{r v}$ either greater or less than $T_{t}$, the dissociation rate will be noticeably affected for even moderate differences. At the most extreme $T_{t}-T_{r v}$ values, the non-equilibrium rate is approximately 300 times greater or less than the equilibrium rate, but these conditions rarely occur in physically realizable flows. At more moderate temperature differences, slices along the surface are insightful, where at $8000 \mathrm{~K}$, if $T_{r v}$ is within $5 \%$ of $T_{t}$ (i.e. $\left|T_{r v}-T_{t}\right|<400 \mathrm{~K}$ ), then the dissociation rate ranges from $75 \%$ to $150 \%$ of the thermal rate. This effect is amplified even further for larger differences. For the temperature range studied, we observe that increasing $T_{r v}$ relative to $T_{t}$ had a more substantial effect on the rate compared to decreasing $T_{r v}$ relative to $T_{t}$ by the same amount. As the temperature increases though, these effects are diminished because the likelihood of dissociation is already high.

Lastly, from these simulations, the present non-equilibrium results are compared to Park's two-temperature model $^{5}$ (with the exception that $T_{r}=T_{v}$ as opposed to $T_{t}$ ), whereby the dissociation rate is given by:

$$
\left.k_{\text {Park }}\left(T_{t}, T_{r v}\right)=k_{e q}\left(\left(T_{t} T_{r v}^{\zeta}\right)^{1 /(1+\zeta}\right)\right),
$$

where $\zeta$ is a constant. Figure 8 shows the rate surface derived using Park's model for a conventional model where $\zeta=1$ and for when $\zeta=4$. 


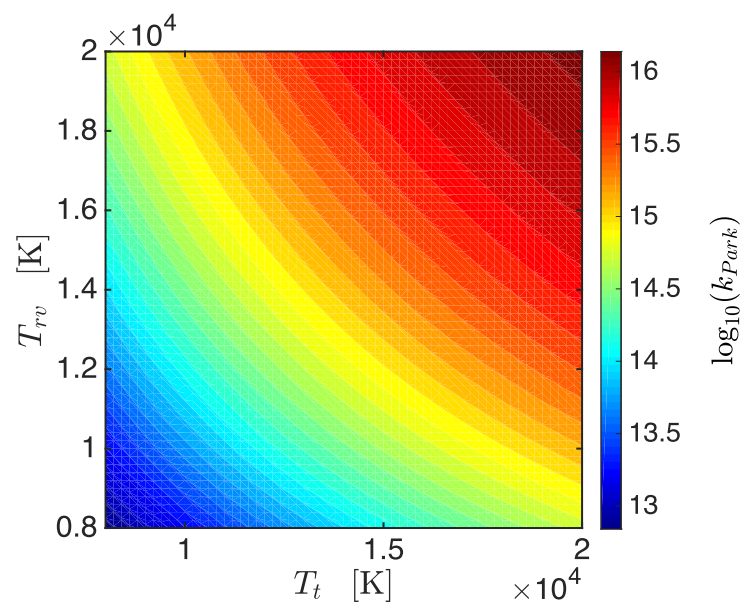

(a)

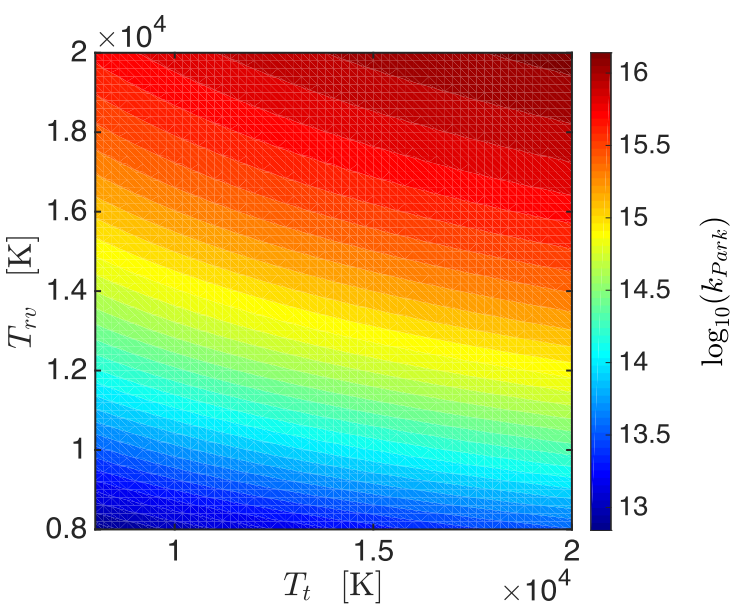

(b)

Figure 8: Dissociation rate versus translational and rovibrational temperature based on Park's twotemperature model: (a) $\zeta=4$, and (b) $\zeta=4$ The units for $k_{d}$ are $\mathrm{cm}^{3} / \mathrm{s} / \mathrm{mol}$.

For $\zeta=1$, by construction the $k_{\text {Park }}$ surface is symmetric across the $T_{t}=T_{r v}$ line, which indicates that an increase in either $T_{t}$ or $T_{r v}$ will have an identical effect on the dissociation rate. This is different from the true non-equilibrium rate as shown in Figure 5a, in which $T_{r v}$ has a larger effect on the rate than $T_{t}$. With this in mind, $\zeta$ was increased to 4 to more closely match the non-equilibrium rate surface. This implies that $T_{r v}$ is approximately four times more influential than $T_{t}$ on the non-equilibrium rate. In the final work, an optimized $m$ and $n$ will be calculated. The efficiency function was also calculated for the Park model when $\zeta=4$. Slices compared the non-equilibrium rate are shown in Figure 9. Even for the non-optimized selection of $\zeta$, this figure validates Park's model for calculating the efficiency function of $\mathrm{N}_{2}$ dissociation. Only for relatively low $T_{t}$ and $T_{r v} \gg T_{t}$, are significant differences between Park's model and the non-equilibrium model observed. It is not yet known if there is an optimal selection of $\zeta$ that minimizes these differences further.

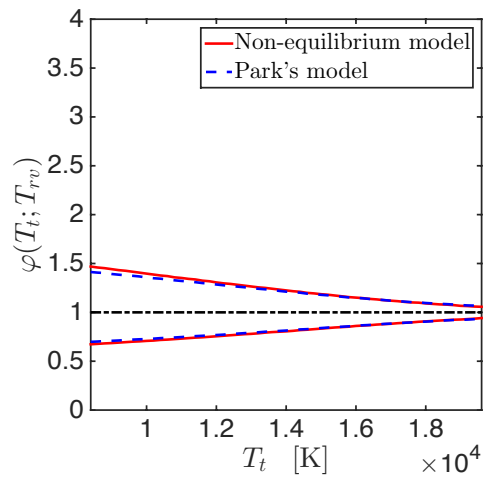

(a)

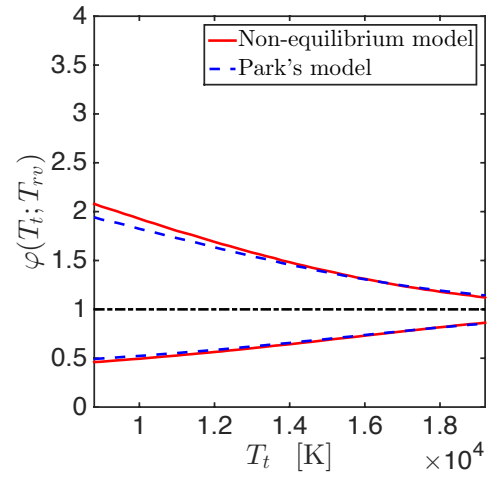

(b)

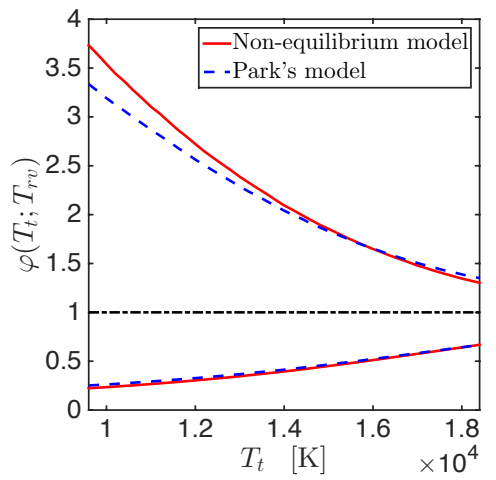

(c)

Figure 9: Slices along the efficiency function surface at various $T_{r v}=T_{t}+\Delta T$ for both the non-equilibrium and Park's model: (a) $T_{r v}=T_{t} \pm 400$, (b) $T_{r v}=T_{t} \pm 800$, and (c) $T_{r v}=T_{t} \pm 1600$ 


\section{Conclusions}

The focus of this work was accurate and efficient calculation of dissociation rates of nitrogen at high temperatures with thermal non-equilibrium. The strategy for accomplishing these goals was to calculate quasi-state-specific dissociation cross-sections and utilize the results to calculate the dissociation rate surface for a large range of translational and rovibrational temperatures. Minimizing and understanding the uncertainty of these calculations was also a critical goal. Specifically, for a fixed total number of trajectories, this work analyzed the consequences of different sampling techniques.

A new massively-parallel QCT program was used to this effect. The features of this code include its ability to scale to many processors while maintaining a high level of efficiency. Using a master/worker core partition strategy, the uncertainty of the directly calculated rates is estimated online throughout the simulation. This allows the number of trajectories for a given state to be truncated after a user-defined tolerance has been achieved. Also, a mode-independent sampling technique was developed so that the impact parameter, relative translational speed, rotational state, and vibrational state of the reactants are all independently sampled.

This first study examined the role of sampling techniques on the dissociation rate uncertainty. It was found that though Monte Carlo sampling poorly estimates low-level information such as the probability of dissociation, it accurately calculates the high-level information such as the dissociation rate. However, the tradeoff for Monte Carlo sampling is that if the state changes, a new set of trajectories must be simulated to calculate the rate. In contrast, uniformly sampling the state leads to a higher uncertainty, but the results can be averaged offline and weighted against a PDF characterized by any temperature. Thus, for CFD applications, uniform sampling is preferable because the reaction rate can be tabulated on a fine-scale grid of temperatures without using interpolation. However, for the rovibrational states, state-specific sampling is computationally intractable to date, so interpolation must be used.

In the second study, a batch of 500 million trajectories was simulated to verify and validate both the newly developed QCT program and the sampling technique's viability. The non-equilibrium rate surface was built using an interpolation strategy that accounted for the uncertainty of the rates. In general, the uncertainty of the surface was small except for low $T_{t}$ and $T_{r v}$. The thermal rate was compared with other models and experimental data, showing strong agreement. The non-equilibrium rate surface showed that $T_{r v}$ has a stronger effect on the dissociation rate than $T_{t}$. This was also observed from the efficiency function of the dissociation rate, where relatively small changes of $T_{r v}$ compared to $T_{t}$ could have a dramatic effect on the rate. Finally, Park's two temperature model was examined and compared to the true non-equilibrium rate. Before calibration, Park's model under-estimated the $T_{r v}$ effect on the dissociation rate compared to $T_{t}$, but after an approximate calibration, the efficiency function of Park's model closely matched the non-equilibrium model.

\section{Acknowledgments}

This work was supported by the AFOSR under grant number FA9550-12-1-0460 with Dr. Ivett Leyva as the grant monitor. The authors thank the Texas Advanced Computing Center (TACC) for their generous allocation of computing time.

\section{References}

\footnotetext{
${ }^{1}$ F. Esposito, I. Armenise, M. Capitelli, $\mathrm{N}-\mathrm{N}_{2}$ state to state vibrational-relaxation and dissociation rates based on quasiclassical calculations, Chemical Physics 331 (1) (2006) 1-8.

${ }^{2}$ Y. Paukku, K. R. Yang, Z. Varga, D. G. Truhlar, Global ab initio ground-state potential energy surface of $\mathrm{N}_{4}$, The Journal of Chemical Physics 139 (4) (2013) 044309.

${ }^{3}$ J. D. Bender, I. Nompelis, P. Valentini, S. Doraiswamy, T. E. Schwartzentruber, G. V. Candler, Y. Paukku, K. R. Yang, Z. Varga, D. G. Truhlar, Quasiclassical trajectory analysis of the $\mathrm{N}_{2}+\mathrm{N}_{2}$ reaction using a new ab initio potential energy surface, 11th AIAA/ASME Joint Thermophysics and Heat Transfer Conference.

${ }^{4}$ A. Munafò, R. L. Jaffe, D. W. Schwenke, M. Panesi, Dissociation and energy transfer study of $\mathrm{N}_{2}-\mathrm{N}_{\text {and }} \mathrm{N}_{2}-\mathrm{N}_{2}$ interactions by using rovibrational and coarse-grained state-to-state models, 53rd AIAA Aerospace Sciences Meeting.

${ }^{5}$ C. Park, Review of chemical-kinetic problems of future nasa missions. I - Earth entries, Journal of Thermophysics and Heat Transfer 7 (3) (1993) 385-398.

${ }^{6}$ R. D. Levine, Molecular Reaction Dynamics, Cambridge University Press, Cambridge, 2005.

${ }^{7}$ J. D. Bender, P. Valentini, I. Nompelis, T. E. Schwartzentruber, G. V. Candler, Characterization of vibrational and rotational energy transfer in $\mathrm{N}_{2}+\mathrm{N}_{2}$ dissociative collisions using the quasiclassical trajectory method, 45th AIAA Thermophysics Conference.
} 
${ }^{8}$ A. Szabo, N. S. Ostlund, Modern Quantum Chemistry: Introduction to Advanced Electronic Structure Theory, Macmillan Publishing Company, New York, 1982.

${ }^{9}$ P. Prince, J. Dormand, High order embedded runge-kutta formulae, Journal of Computational and Applied Mathematics 7 (1) (1981) 67-75.

${ }^{10}$ W. L. Hase, R. J. Duchovic, X. Hu, A. Komornicki, K. F. Lim, D.-H. Lu, G. H. Peslherbe, K. N. Swamy, S. R. V. Linde, A. Varandas, H. Wang, R. J. Wolf, Venus96: A general chemical dynamics computer program, Quantum Chemistry Program Exchange 16 (671).

${ }^{11}$ Q. Wang, P. Moin, G. Iaccarino, A high order multivariate approximation scheme for scattered data sets, Journal of Computational Physics 229 (18) (2010) 6343-6361.

${ }^{12}$ R. K. Hanson, D. Baganoff, Shock-tube study of nitrogen dissociation rates using pressure measurements, AIAA Journal 10 (2) (1972) 211-215.

${ }^{13}$ O. Knab, H.-H. Fruehauf, E. W. Messerschmid, Theory and validation of the physically consistent coupled vibrationchemistry-vibration model, Journal of Thermophysics and Heat Transfer 9 (2) (1995) 219-226. 